\title{
Graphene transparent electrodes grown by rapid chemical vapor deposition with ultrathin indium tin oxide contact layers for GaN light emitting diodes
}

\author{
Xu Kun, ${ }^{1}$ Xu Chen, ${ }^{1, a)}$ Deng Jun, ${ }^{1}$ Zhu Yanxu, ${ }^{1}$ Guo Weiling, ${ }^{1}$ Mao Mingming,,${ }^{1}$ Zheng Lei, ${ }^{1}$ \\ and Sun Jie $1,2, \mathrm{~b})$ \\ ${ }^{1}$ Key Laboratory of Optoelectronics Technology (Beijing University of Technology), Ministry of Education, \\ Beijing 100124, China \\ ${ }^{2}$ Mikroteknologi och Nanovetenskap, Chalmers Tekniska Högskola AB, Göteborg 41296, Sweden
}

(Received 1 January 2013; accepted 9 April 2013; published online 22 April 2013)

\begin{abstract}
By virtue of the small active volume around $\mathrm{Cu}$ catalyst, graphene is synthesized by fast chemical vapor deposition (CVD) in a cold wall vertical system. Despite being highly polycrystalline, it is as conductive and transparent as standard graphene and can be used in light emitting diodes as transparent electrodes. $7-10 \mathrm{~nm}$ indium tin oxide (ITO) contact layer is inserted between the graphene and $p-\mathrm{GaN}$ to enhance hole injection. Devices with forward voltage and transparency comparable to those using traditional $240 \mathrm{~nm}$ ITO are achieved with better ultraviolet performances, hinting the promising future for application-oriented graphene by rapid CVD. (C) 2013 AIP Publishing LLC [http://dx.doi.org/10.1063/1.4802798]
\end{abstract}

Graphene, a truly two-dimension material, has attracted extensive attention worldwide due to its superior properties such as high electrical conductivity and optical transmission. For some time, however, the use of graphene is severely limited by its synthesis methods: small size (mechanical exfoliation), ${ }^{1}$ defective structure (liquid phase exfoliation), ${ }^{2}$ or high cost (SiC epitaxy). ${ }^{3}$ Recently, chemical vapor deposition (CVD) has shown to be an industry compatible method capable of producing scalable monolayer graphene with properties suitable for electronic applications. ${ }^{4,5}$ It is forecasted to be commercialized as transparent conductive layers (TCLs) in optoelectronic devices in a few years. ${ }^{6}$ Indium tin oxide (ITO), presently dominant for transparent electrodes, suffering from low transmission in ultraviolet region, ever increasing price due to In scarcity, low flexibility, and poor stability in harsh chemical environments, is expected to be substituted by graphene. Nevertheless, for these claims to become reality, the cost of graphene CVD is yet to be reduced to be competitive in market. Currently, there is a trend in the CVD community to pursue large graphene single crystals. For instance, sophisticated electrochemical polishing of $\mathrm{Cu}, 7 \mathrm{~h}$ annealing $\left(1077^{\circ} \mathrm{C}\right)$ and $2 \mathrm{~h}$ deposition are required to boost the crystal size to $4.5 \mathrm{~mm}^{2}$. Still far from wafer size, the deposition is already orders of magnitude slower than semiconductor epi$\operatorname{taxy}^{8}$ in order to reduce nucleation density, implying an unaffordable high consumption of energy and time for industry. Thus, while being vital for fundamental research, this route is hardly valuable towards applications. In this letter, we argue for the opposite: fast CVD using a cold wall vertical system, where a complete run is done within $0.5 \mathrm{~h}$, yielding uniform graphene films with $\mu \mathrm{m}$-sized crystallites.

Can this kind of graphene meet the stringent criteria of high-end electronic applications? Recently, Tsen et al. observed that graphene with greater polycrystallinity unexpectedly yielded highly uniform coverage of the $\mathrm{Cu}$ surface. ${ }^{9}$ Grain boundaries grown under optimized conditions have

\footnotetext{
${ }^{\text {a)} E l e c t r o n i c ~ m a i l: ~ x u c h e n 58 @ b j u t . e d u . c n ~}$

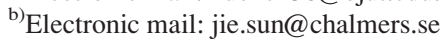

good stitching and, therefore, induce minimal negative impact on electron transport. ${ }^{9}$ Sun et al. demonstrated that graphene with nice interdomain connectivity was equally transparent as single crystalline graphene. ${ }^{10,11}$ In this letter, employing GaN light emitting diodes (LEDs) as example devices, we elaborate on the application of our graphene grown by rapid CVD in optoelectronics. GaN-based LEDs are core components for next generation lighting. But impurities in $p$-GaN are difficult to ionize, and the current expansion ability is limited due to its low hole density. We have used graphene as the current spreading layer to accomplish high performance GaN LEDs. Graphene's low work function was compensated by using ultrathin ITO $(7-10 \mathrm{~nm})$ as contact layer to GaN to improve hole injection. Previous graphene-only devices suffered from high forward voltage $\left(V_{f}>10 \mathrm{~V}\right) .{ }^{12}$ Other solutions include using interlayers of thin metal $^{13,14}$ or ITO nanodots. ${ }^{15}$ Nevertheless, these attempts were all at the expense of losing transparency (e.g., merely $78 \%$ for $1 \mathrm{~nm} \mathrm{Ni} / 1 \mathrm{~nm} \mathrm{Au}$ /monolayer graphene). ${ }^{14}$ We note that optical transmission is ultra important for LEDs. Poor transmission not only directly lowers the output power but also transforms the absorbed light to heat, further decreasing internal quantum efficiency. Moreover, process control to form extremely thin and uniform metal sheets or proper nanodots by annealing and etching $250 \mathrm{~nm}$ thick ITO is not trivial. In this letter, our ITO nanolayer significantly improves the electrical contact between graphene TCL and $p$-GaN and brings the operating voltage $\left(V_{f}=3.9 \mathrm{~V}\right.$ at $20 \mathrm{~mA}$ input current) down to that of conventional LEDs with $240 \mathrm{~nm}$ ITO TCL. The addition of ITO nanolayer has negligible optical absorption $(99.4 \%$ at $460 \mathrm{~nm}$, higher than that of previous transition layers). This structure has not been reported in literature, where we have obtained low $V_{f}$ and high transmission simultaneously. Meanwhile, this is a facile method with low cost, compatible to the mainstream LED technology. It hints the promising future of commercialization of graphene synthesized by fast CVD.

The graphene was deposited on polycrystalline $\mathrm{Cu}$ foils placed on a Joule heater of a vertical cold wall CVD 


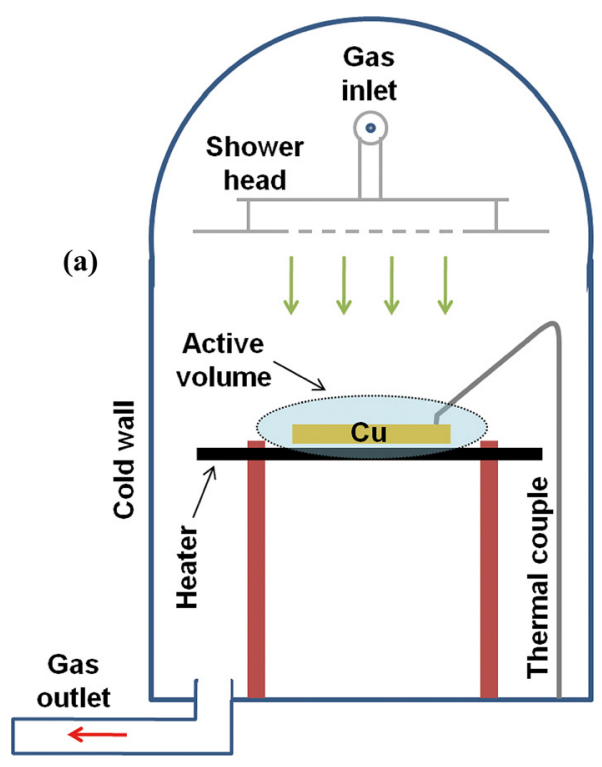

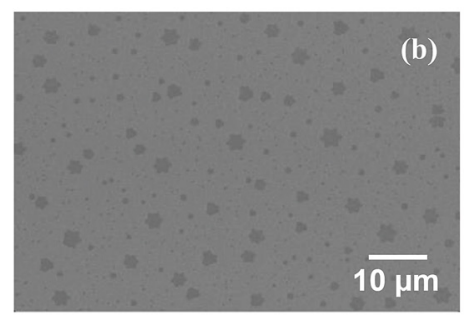

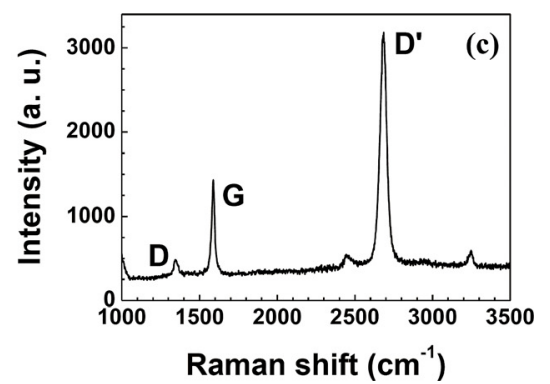

FIG. 1. (a) Schematic drawing of the cold wall CVD. Gases are injected from the shower head over the sample sitting on a heater whose $T$ is measured by a thermal couple. Most of the distance that the gas species travel before they reach the $\mathrm{Cu}$ is cold, which is equivalent to elongated gas line within the growth chamber, resulting in a very small active hot volume indicated by dashed line, as opposed to situations in hot wall systems. (b) SEM image of the high density graphene nucleation during the early stage deposition on liquid $\mathrm{Cu}$, captured after cooling to room temperature. (c) Typical Raman spectrum of the graphene. $\mathrm{D}^{\prime}$ intensity is more than twice higher than $G$. The small D peak implies the high quality of the film.
(Aixtron) as shown in Fig. 1(a). After organic cleaning, the sample was rapidly heated to $1000^{\circ} \mathrm{C}\left(\operatorname{ramp}>300^{\circ} \mathrm{C} / \mathrm{min}\right)$ and annealed for $5 \mathrm{~min}$. The growth was done for $5 \mathrm{~min}$ in $1.5 \mathrm{sccm} \mathrm{CH}_{4}, 20 \mathrm{sccm} \mathrm{H}_{2}$, and $1030 \mathrm{sccm} \mathrm{Ar}(P=6.35 \mathrm{mbar})$ followed by quench cooling (in the same gas flow) by cutting off the heater power. The full process was finished within $30 \mathrm{~min}$. The epitaxial wafer used in this experiment was a commercial structure consisting of undoped $(u)-\mathrm{GaN}$, $n$-GaN, InGaN quantum wells and $p$-GaN grown by metalorganic CVD on $c$-plane (0001) sapphire substrate. Five types of TCL structures were fabricated in the GaN-based LEDs $(10 \mathrm{mil} \times 16 \mathrm{mil}$ in size, $1 \mathrm{mil}=25.4 \mu \mathrm{m})$. Type I: $240 \mathrm{~nm}$ ITO (standard TCL for $p$-GaN); type II: 3-layer graphene; type III, IV, and V: 3, 7, and $10 \mathrm{~nm}$ ITO + 3-layer graphene, respectively (see Fig. 2 for the structure diagram). The device processing is as follows. After organic cleaning, the $\mathrm{GaN}$ wafer was boiled in aqua regia to remove native oxides and metal contamination and rinsed with deionized water.

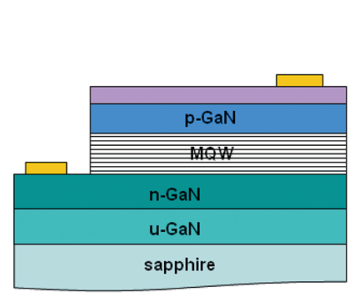

(a)

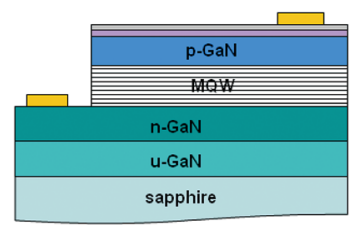

(c)

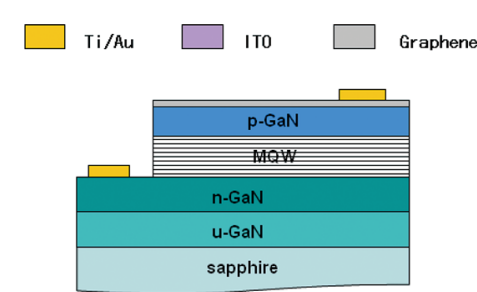

(b)

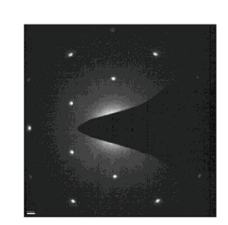

(d)
FIG. 2. Structures of (a) type I (240 nm ITO), (b) type II (3-layer graphene), and (c) type III, IV, and V (3, 7, and $10 \mathrm{~nm}$ ITO + 3-layer graphene) TCLs used in GaN LEDs. MQW denotes multiple quantum wells. (d) Typical selected area electron diffraction pattern of the graphene. Six-fold symmetric dots with equal intensity strength clearly indicate high quality monolayer graphene.
The ITO was deposited by electron beam evaporation. The graphene was transferred layer by layer via standard wet etching of $\mathrm{Cu}$ in $\mathrm{FeCl}_{3}$ aqueous solution, ${ }^{5}$ using spin coated poly(methyl methacrylate) (PMMA) mechanical support. Mesa areas were defined by photolithography, where the graphene and/or ITO nanolayers outside the mesa areas were removed by oxygen plasma and/or ITO etchant. Then, the gallium nitride was etched $1.1-1.2 \mu \mathrm{m}$ deep via inductively coupled plasma etching down to $n$-GaN. Finally, patterned metal electrodes $(15 \mathrm{~nm} \mathrm{Ti} / 300 \mathrm{~nm} \mathrm{Au})$ were fabricated by photolithography and sputtering.

Key factors for the fast graphene CVD are explained as follows. Unlike common hot wall quartz tube furnaces where everywhere is isothermal, here only the heater is hot up by AC Joule current. Therefore, the sample can be heated to $1000^{\circ} \mathrm{C}$ in $3 \mathrm{~min}$. The gas flow comes from right above, rendering a "head-on collision" situation, where most gas molecules have chances to meet the catalyst. In horizontal tubes, however, the gases and the sample are often like "large angle scattering." Only a skinny layer of gas molecules can interact with the sample whereas the majority is wasted. Therefore, our geometry offers the most efficient use of the precursor and the deposition can be done at a partial pressure of only $P_{\text {methane }}=0.009$ mbar. Most importantly, our quick deposition is ensured by the extremely small active volume (Fig. 1(a)). In graphene CVD, two sequential reactions take place: $\mathrm{CH}_{4} \leftrightarrow \mathrm{C}+2 \mathrm{H}_{2}$ and $6 \mathrm{C} \leftrightarrow \mathrm{C}_{6}$. For the $\mathrm{Cu}$ catalyst to effectively push the chemical equilibrium point of the second equation forward, according to Le Chatelier's principle, the concentration of reactant ( $\mathrm{C}$ in the active volume) should be high enough. In other words, a large size of $\mathrm{Cu}$ and a narrow active space around the sample are helpful for complete decomposition of hydrocarbon and graphitization. In a cold wall system, the heating mechanism is dominantly radiation and gas conduction, ${ }^{16}$ which both strongly depend on the solid angle (hence the distance to heater). Therefore, only a small volume around the sample is hot and active (Fig. 1(a)), leading to the high speed of deposition. We have also grown graphene on molten $\mathrm{Cu}$ (Ref. 17) $\left(>1200^{\circ} \mathrm{C}\right.$ ) in the same flow/pressure for $5 \mathrm{~min}$ (incomplete coverage due to the 


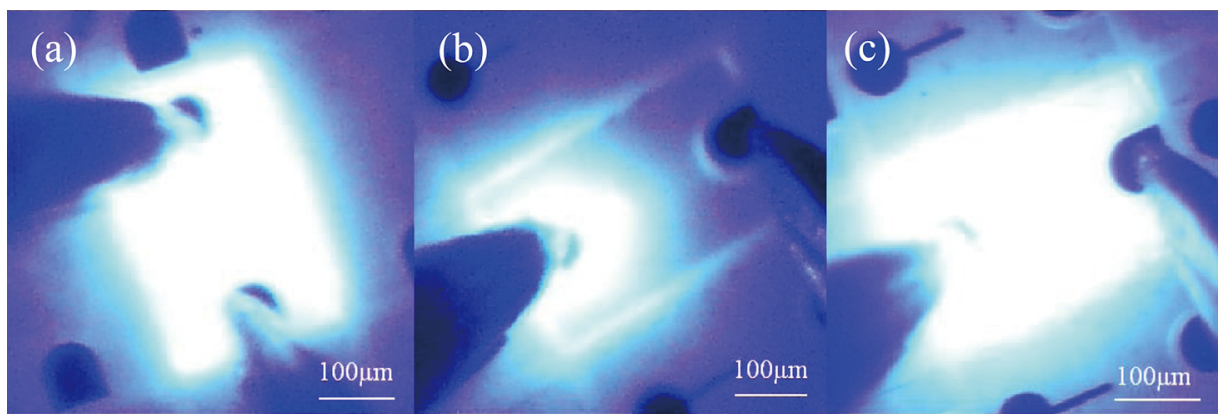

FIG. 3. Optical graphs of the electroluminescence from GaN blue LEDs using (a) type II (3-layer graphene), (b) $10 \mathrm{~nm}$ ITO only, and (c) type V (10 nm ITO + 3-layer graphene) TCLs. Evidently, the current in (a) and (c) is uniformly distributed across the mesas.

lower deposition rate at higher $T$ ). Indeed, from the scanning electron microscopy (SEM) image in Fig. 1(b), the nucleation density is $>0.1 \mu \mathrm{m}^{-2}$. Naturally, for solid Cu with much rougher surface and lower $T$, the nucleation and growth should be even faster, resulting in well-stitched $\mu \mathrm{m}$-sized crystallites with full coverage on $\mathrm{Cu}$.

As pointed out by Tsen et al., ${ }^{9}$ despite the highly polycrystalline nature, optimized growth will lead to good transport properties. The as-deposited graphene shows sheet resistance $R_{s} 800-1000 \Omega / \square$ for monolayer and $300-350 \Omega / \square$ for three layers, in the same order as standard graphene. ${ }^{18}$ Raman spectrum in Fig. 1(c) and electron diffraction pattern by transmission electron microscopy in Fig. 2(d) confirm the high lattice quality of the monolayer graphene. Hall measurement reveals a carrier mobility of $\sim 2000 \mathrm{~cm}^{2} / \mathrm{Vs}$ at room temperature in air (no annealing). ${ }^{19}$ Fig. 3 shows photos of the light emission effect for three types of TCLs: (a) 3-layer graphene, (b) $10 \mathrm{~nm}$ ITO, and (c) $10 \mathrm{~nm}$ ITO + 3-layer graphene applied in the GaN blue LED devices at $20 \mathrm{~mA}$ input current. In Fig. 3(a), the luminescence from the whole device is uniform, revealing that the graphene TCL spreads current well. We note that the adhesion between graphene and $\mathrm{GaN}$ is generally good (compared with the case of graphene/GaAs, etc.), probably due to the lattice similarity induced affinity. Fig. 4(a) is the SEM image of a control sample, where basically no holes and wrinkles in the single layer graphene are detected on GaN after transfer, except for a few bilayer flakes. In Fig. 3(b), the luminous area of the LED is limited to the $p$-pad adjacency, indicating $10 \mathrm{~nm}$ ITO cannot meet the requirement for current expansion. In Fig. 3(c), the light emission is again as uniform as (a), confirming the essential role of graphene.

Fig. 4(c) summarizes the $I-V$ curves of the GaN-based LEDs with five kinds of TCLs. At $20 \mathrm{~mA}$, high operating voltage $(6.76 \mathrm{~V})$ was observed in graphene-only TCL due to the big work function mismatch between graphene $(\Phi=4.5 \mathrm{eV})$ and $p-\mathrm{GaN}(\Phi=7.5 \mathrm{eV})$. The barrier in the contacts is obviously high. This problem is addressed by inserting ITO nanolayers between $p-\mathrm{GaN}$ and graphene. In device IV, the $I-V$ properties improve dramatically, with $V_{f}=3.90 \mathrm{~V}$ at $20 \mathrm{~mA}$, only $0.3 \mathrm{~V}$ higher than device I (standard $240 \mathrm{~nm}$ ITO TCL). ITO has better contact to $p$-GaN than graphene largely because of its greater work function $(\Phi=4.8 \mathrm{eV})$ and cleaner interface. Further increasing ITO thickness to $10 \mathrm{~nm}$ (type V) gives similar result as IV, whereas $3 \mathrm{~nm}$ (type III) is apparently insufficient to attain nice $I-V$ properties. To understand the yet present discrepancy between the $V_{f}$ of structures IV, $\mathrm{V}$, and I, we plot the $I-V$ curves of Ti/Au-ITO-Ti/Au and Ti/Au-graphene-ITO-graphene-Ti/Au junctions in Fig. 4(d), where the structures are schematically shown in Fig. 4(b). Here the graphene has 3 layers, and the thickness of ITO is $240 \mathrm{~nm}$. The identical curves in Fig. 4(d) strongly suggest that the contact resistances of graphene/metal and graphene/ITO
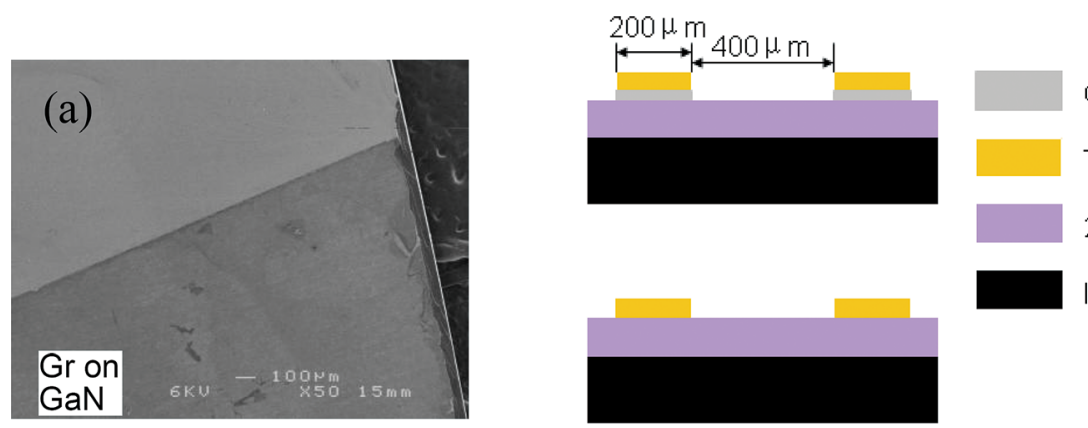

Graphene

Ti/ALU

$240 \mathrm{~nm}$ ITO

Insulator

(b)
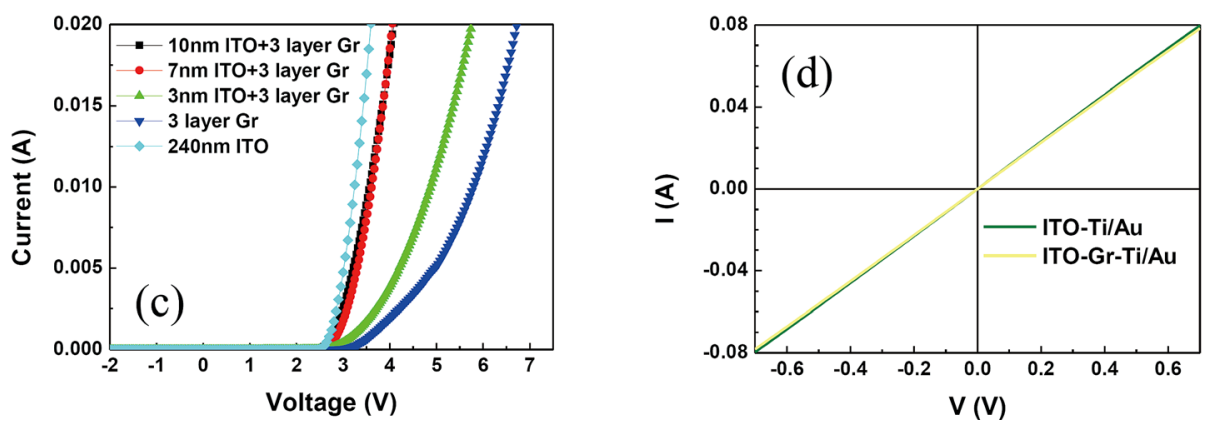

FIG. 4. (a) Typical SEM micrograph of the as-grown graphene wet transferred to $\mathrm{GaN}$ substrate (control sample). The upper part is uncovered. (b) Geometry of the two test structures used for the measurements in (d). (c) $I-V$ characteristics of the fabricated $\mathrm{GaN}$ LEDs with assorted TCLs as indicated by the text. (d) Two-terminal $I-V$ curves recorded between the electrodes shown in (b). Gr denotes graphene. 


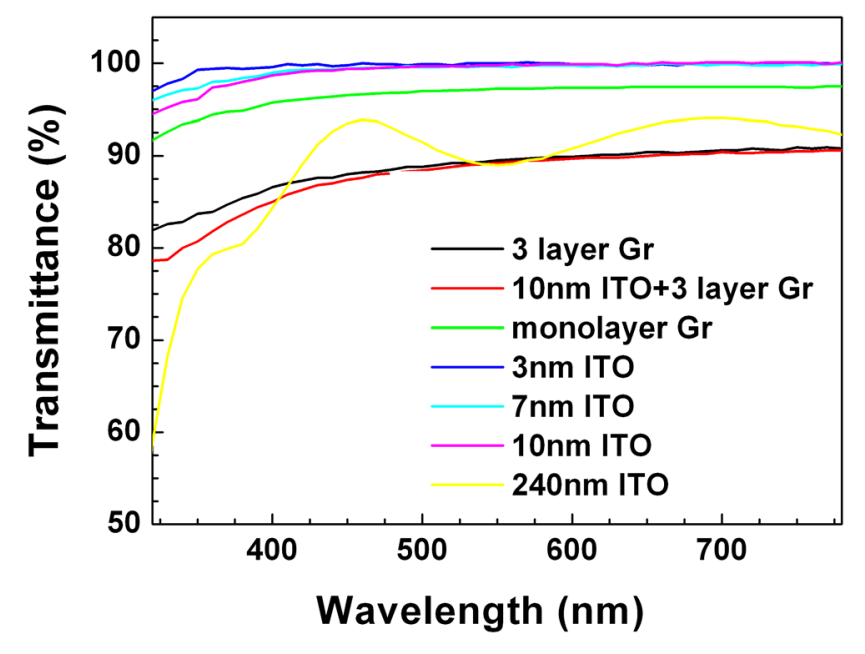

FIG. 5. Comparison of the optical transmittance properties of several TCLs. Towards the ultraviolet regime, ITO is increasingly opaque. Gr denotes graphene.

are exceedingly small. Therefore, the difference between the $V_{f}$ of structures IV, V, and I can only be ascribed to the sheet resistance: $R_{s}$ for $240 \mathrm{~nm}$ ITO is merely $\sim 30 \Omega / \square$. Note that the graphene employed in this experiment did not undergo any intentional doping, because we found that the chemical modification effect (e.g., by $\left.\mathrm{AuCl}_{3}\right)^{20}$ was not stable with time. Future technical breakthroughs in graphene $p$-doping are expected to increase $\Phi$ to a level that its sheet resistance and contact resistivity to $p$-GaN can be both significantly reduced. Even so, however, the graphene-GaN interface might still lack the quality required to bring $V_{f}$ to the same level as conventional ITO-based GaN LEDs. One solution is to directly grow carbon on $\mathrm{GaN}^{21}$ where the interface is much cleaner and Schottky barriers are not observed, hence possibly thoroughly leave out ITO.

Fig. 5 shows a comparison of the optical transmission of $240 \mathrm{~nm}$ standard ITO, various thickness ITO transition layers, 1- and 3-layer graphene, and $10 \mathrm{~nm} \mathrm{ITO+3-layer}$ graphene. Obviously, the transmission of $3 \mathrm{~nm}, 7 \mathrm{~nm}$, and $10 \mathrm{~nm}$ ITO is very high, nearly $100 \%$ in $550 \mathrm{~nm}-780 \mathrm{~nm}$ wavelength region, slightly descendent for $<550 \mathrm{~nm}$, but still quite high compared to other transition layers such as $\mathrm{Au}$ or $\mathrm{Ni} / \mathrm{Au}$ sheets. $^{13,14}$ Typically, the transmissions of $3 \mathrm{~nm}$, $7 \mathrm{~nm}$, and $10 \mathrm{~nm} \mathrm{ITO}$ are $99.9 \%, 99.6 \%$, and $99.4 \%$ at $460 \mathrm{~nm}$ and $97 \%, 96 \%$, and $94.5 \%$ at $320 \mathrm{~nm}$, respectively. At $460 \mathrm{~nm}$ and $320 \mathrm{~nm}$, monolayer (trilayer) graphene has transparencies of $96.7 \%(88.2 \%)$ and $91.7 \%(81.9 \%)$, respectively. These data correspond well with theoretical values. ${ }^{18}$ It is seen that the transmission of type V TCL $(10 \mathrm{~nm}$ ITO +3 -layer graphene) is very close to that of 3-layer graphene. Compared with $240 \mathrm{~nm}$ ITO, while possessing a generally comparable transmission, in ultraviolet region (e.g., $320 \mathrm{~nm}$ ), type V TCL is considerably more transparent (78.6\% vs. $58.9 \%)$. Our results indicate that $7-10 \mathrm{~nm}$ ITO interface layers, identified as the optimal parameters, are enough to lower the operating voltage of GaN LED with graphene TCL effectively. Further increase of the thickness of ITO has little improvement on $V_{f}$. Rather, the transmission is degraded instead. Type III TCL $(3 \mathrm{~nm}$ ITO + 3-layer graphene), however, can only moderately improve the LED $I-V$ characteristics, due to the fact that too thin a thickness in
ITO inevitably leads to grainy island structures, limiting the enhancement effect on the hole injection from the graphene to $p-\mathrm{GaN}$.

In summary, contrary to many groups pursuing $\mathrm{mm}$ sized "large crystals" grown by slow processes, we suggest a facile synthesis of graphene by rapid CVD within $0.5 \mathrm{~h}$. The as-deposited material has crystallites in $\mu \mathrm{m}$ size, showing electrical conductivity and optical transparency similar to standard graphene and can be achieved at much more reduced energy and time consumption. As a proof-ofconcept experiment, we are affording a method for applying the graphene in GaN-based LEDs as current spreading layer. Innovatively, the ITO nanolayers $(7-10 \mathrm{~nm})$ employed in this experiment help to overcome the contact barrier between graphene and $p$-GaN and, meanwhile, keep the overall transmission almost unaffected, which is apparently very useful to improve the output power of LEDs. On the other hand, earlier studies either suffered from high $V_{f}$ or unsatisfactory transmission due to inappropriate interlayers. The material and device processes reported here are facile methods with high reproducibility, very promising for the commercial application in the future. At this stage, stable and effective doping of nanocarbon structures has not been established worldwide. Notwithstanding, the overall performance of the graphene-based TCLs is already comparable to that of $240 \mathrm{~nm}$ ITO which is rivaled in the ultraviolet regime. Our application-oriented results point at the promising future of the graphene grown by fast CVD, which would eventually replace ITO as TCLs in optoelectronics.

This work was supported by the National Natural Science Foundation of China (Grant Nos. 61076044, 61107026, and 61204011), the Natural Science Foundation of Beijing, China (Grant Nos. 4132006, 4102003, and 4112006), the Specialized Research Fund for the Doctoral Program of Higher Education of China (Grant No. 20121103110018), the National High Technology Research and Development Program of China (Grant No. 2008AA03Z402), and the Special Grant for BJUT LargeArea Layer-Controllable Graphene Carbon Nanoelectronics Teaching and Research Base.

${ }^{1}$ K. S. Novoselov, A. K. Geim, S. V. Morozov, D. Jiang, Y. Zhang, S. V. Dubonos, I. V. Grigorieva, and A. A. Firsov, Science 306, 666 (2004).

${ }^{2}$ Y. Hernandez, V. Nicolosi, M. Lotya, F. M. Blighe, Z. Sun, S. De, I. T. McGovern, B. Holland, M. Byrne, Y. K. Gun'Ko, J. J. Boland, P. Niraj, G. Duesberg, S. Krishnamurthy, R. Goodhue, J. Hutchison, V. Scardaci, A. C. Ferrari, and J. N. Coleman, Nat. Nanotechnol. 3, 563 (2008).

${ }^{3}$ A. Tzalenchuk, S. Lara-Avila, A. Kalaboukhov, S. Paolillo, M. Syväjärvi, R. Yakimova, O. Kazakova, T. J. B. M. Janssen, V. Fal'ko, and S. Kubatkin, Nat. Nanotechnol. 5, 186 (2010).

${ }^{4}$ S. Bae, H. Kim, Y. Lee, X. Xu, J.-S. Park, Y. Zheng, J. Balakrishnan, T. Lei, H. R. Kim, Y. I. Song, Y.-J. Kim, K. S. Kim, B. Özyilmaz, J.-H. Ahn, B. H. Hong, and S. Iijima, Nat. Nanotechnol. 5, 574 (2010).

${ }^{5}$ J. Sun, N. Lindvall, M. T. Cole, K. T. T. Angel, T. Wang, K. B. K. Teo, D. H. C. Chua, J. Liu, and A. Yurgens, IEEE Trans. Nanotechnol. 11, 255 (2012).

${ }^{6}$ K. S. Novoselov, V. I. Fal'ko, L. Colombo, P. R. Gellert, M. G. Schwab, and K. Kim, Nature (London) 490, 192 (2012).

${ }^{7}$ Z. Yan, J. Lin, Z. Peng, Z. Sun, Y. Zhu, L. Li, C. Xiang, E. L. Samuel, C. Kittrell, and J. M. Tour, ACS Nano 6, 9110 (2012).

${ }^{8}$ J. Sun, P. Jin, and Z.-G. Wang, Nanotechnology 15, 1763 (2004).

${ }^{9}$ A. W. Tsen, L. Brown, M. P. Levendorf, F. Ghahari, P. Y. Huang, R. W. Havener, C. S. Ruiz-Vargas, D. A. Muller, P. Kim, and J. Park, Science 336, 1143 (2012). 
${ }^{10}$ J. Sun, M. T. Cole, N. Lindvall, K. B. K. Teo, and A. Yurgens, Appl. Phys. Lett. 100, 022102 (2012).

${ }^{11}$ J. Sun, N. Lindvall, M. T. Cole, K. B. K. Teo, and A. Yurgens, Appl. Phys. Lett. 98, 252107 (2011).

${ }^{12}$ B.-J. Kim, C. Lee, Y. Jung, K. H. Baik, M. A. Mastro, J. K. Hite, C. R. Eddy, Jr., and J. Kim, Appl. Phys. Lett. 99, 143101 (2011).

${ }^{13}$ S. Chandramohan, J. H. Kang, Y. S. Katharria, N. Han, Y. S. Beak, K. B. Ko, J. B. Park, H. K. Kim, E.-K. Suh, and C.-H. Hong, Appl. Phys. Lett. 100, 023502 (2012).

${ }^{14}$ J. M. Lee, H. Y. Jeong, K. J. Choi, and W. I. Park, Appl. Phys. Lett. 99, 041115 (2011).

${ }^{15}$ T. H. Seo, K. J. Lee, T. S. Oh, Y. S. Lee, H. Jeong, A. H. Park, H. Kim, Y. R. Choi, E.-K. Suh, T. V. Cuong, V. H. Pham, J. S. Chung, and E. J. Kim, Appl. Phys. Lett. 98, 251114 (2011).
${ }^{16}$ A. Hasper, J. E. J. Schmitz, J. Holleman, and J. F. Verwey, J. Vac. Sci. Technol. A 10, 3193 (1992).

${ }^{17}$ D. Geng, B. Wu, Y. Guo, L. Huang, Y. Xue, J. Chen, G. Yu, L. Jiang, W. Hu, and Y. Liu, Proc. Natl. Acad. Sci. U.S.A. 109, 7992 (2012).

${ }^{18}$ F. Bonaccorso, Z. Sun, T. Hasan, and A. C. Ferrari, Nature Photon. 4, 611 (2010).

${ }^{19}$ C. J. L. Rosa, J. Sun, N. Lindvall, M. T. Cole, Y. Nam, M. Löffler, E. Olsson, K. B. K. Teo, and A. Yurgens, Appl. Phys. Lett. 102, 022101 (2013).

${ }^{20}$ K. K. Kim, A. Reina, Y. Shi, H. Park, L.-J. Li, Y. H. Lee, and J. Kong, Nanotechnology 21, 285205 (2010).

${ }^{21}$ J. Sun, M. T. Cole, S. A. Ahmad, O. Bäcke, T. Ive, M. Löffler, N. Lindvall, E. Olsson, K. B. K. Teo, J. Liu, A. Larsson, A. Yurgens, and Å. Haglund, IEEE Trans. Semicond. Manuf. 25, 494 (2012). 